\title{
EDITORIAL
}

\section{Unfolding the mechanisms of progression of pulmonary emphysema in COPD}

\author{
Riccardo Pellegrino and Andrea Antonelli
}

$\mathbf{U}$ ntil a quarter of a century ago, diagnosing pulmonary emphysema in clinical practice was a very difficult task, as the definition of the disease was based only on the pathological criteria of destruction of the parenchyma distal to the terminal bronchioles in the absence of inflammation and/or fibrosis [1]. Lung function tests could be of help but this was only possible when airflow obstruction was severe, lung volumes increased above normal and diffusing capacity of the lung very low, i.e. too late to identify the disease in the early stages. Similarly, chest radiography could be of help for this purpose but only when lung fields were hyperlucent with blebs and no or few arteries, the diaphragm was flat, and anteroposterior diameters were increased. Again, this was when the disease was too advanced. A major breakthrough occurred with the advent of high-resolution computed tomography (HRCT), a tool that proved to be very sensitive in assessing the pathology on its centrilobular, panlobular and paraseptal features, and its distribution across the lungs $[2,3]$. The results were so impressive that the technique was soon widely used in both clinical practice and research. In addition to assisting in the clinical diagnosis of the disease condition, HRCT brought major advancements as for the pathogenesis of the chronic obstructive pulmonary disease (COPD), and nowadays plays a crucial role with respect to lung volume reduction surgery [4] and transplantation [5].

Recent studies have focused on HRCT as a tool to assess the progression of the disease. Most of them have been conducted in $\alpha_{1}$-antitrypsin deficiency with the aim of identifying the lung densitometry parameters that best reflect the progression of the disease [6] compared with classical functional parameters, such as forced expiratory volume in $1 \mathrm{~s}$ (FEV1) and diffusing capacity of the lung for carbon monoxide (DL,CO) [7], or health status parameters [8]. Interestingly, the Hounsfield unit point below which $15 \%$ of the low attenuation voxels were distributed (Perc15) and densitometry of the computed tomogram emerged as the most sensitive parameters to assess the temporal and spatial evolution of the disease together with classical functional parameters such as FEV1 and DL,CO, depending on the stage and topographical distribution of the disease $[7,9]$. In addition to these findings, these studies had the merit of opening new horizons in the understanding of the natural course of the disease.

Allergologia e Fisiopatologia Respiratoria, Azienda Ospedaliera S. Croce e Carle, Cuneo, Italy.

CORRESPONDENCE: R. Pellegrino, Allergologia e Fisiopatologia Respiratoria, Azienda Ospedaliera S. Croce e Carle, 12100 Cuneo, Italy. E-mail: pellegrino.r@ospedale.cuneo.it
What we know today is that emphysema originates in the lung as a result of a series of mechanisms variably contributing to cause destruction of parenchymal tissue distal to the terminal bronchioles. Among these mechanisms, protease-antiprotease imbalance, oxidative stress, inflammation, apoptosis and matrix remodelling are on top of the list [10]. Yet, it is striking that even if the original causes are removed, the disease still progresses and leads to severe destruction of the lung and, eventually, respiratory insufficiency. To explain such a paradox, it has been proposed that the progression of disease and decline of lung function are sustained by mechanical factors [11, 12]. Basically, the idea is that after being exposed to proteases, oxidants and other chemical compounds, alveolar units may become prone to breaking as a result of the stretching imposed by breathing [13]. Evidence for this comes from a series of studies and/or observations. For instance, emphysema in smokers is predominantly distributed within the upper lung regions where the alveolar units are subjected to a greater stress than their gravitydependent counterpart [14]. In a rat model of elastase-induced emphysema, the threshold for mechanical failure of collagen has been found to be reduced, thus suggesting that mechanical forces of ventilation have the potential to disrupt extracellular matrix [15]. Similar deductions can be inferred from the faster deterioration of lung function observed after lung volume reduction surgery compared with before the intervention [16]. Presumably, with redistribution of mechanical forces throughout the lung, alveolar regions exposed to larger pressures can now overinflate again and progressively break up, thus contributing to perpetuate the original disease. A recent very elegant model proposed by WINKLER and SUKI [13] helps understand the step-by-step the progression of the disease. In a network with identical elastic and linear springs under slight tension, it is sufficient that if one breaks down, the force is redistributed to the other springs that become now exposed to greater tension. At the same time, some of the spaces enlarge. With further breaks of springs, the phenomenon replicates, thus leading to new configurations, each of them with increased number and dimension of holes. Turning to the lung, it is like the lung parenchyma would flake off after the initial insult as a result of the continuous stretching imposed by breathing. In the centrilobular pattern, the preferential distribution of emphysema to the upper lobes could thus be the result of the low pleural pressure acting on the alveolar regions previously exposed to smoke or pollution, whereas in the panlobular pattern the more homogeneous distribution along the vertical axis could be the result of a local imbalance between $\alpha_{1}$ antitrypsin and elastase. 
In this context, a study by MOHAMED HoEsein et al. [17] published in this issue of the European Respiratory Journal is of great import and corroborates the notion that upper lobe emphysema contributes to accelerating the lung function decline. The authors took advantage of the NELSON study in which 587 smokers had lung computed tomography scanning and lung function measured at baseline and about 3 yrs later. After anatomical segmentation of each lung into lobes, emphysema was assessed as Perc15. Using principal component analysis, estimates of total lung emphysema severity and emphysema distribution were highly correlated with the decrease in FEV1, forced vital capacity (FVC) and FEV1/FVC ratio after 3 yrs, and this was true for either former or current smokers. The impressive number of participants in the study, the longitudinal design of the study and the ability of the authors to segment into lobes rather than fractions of lungs lend strong support to the hypothesis that it is within the upper lobe emphysematous regions that lung function declines rapidly in smokers. Given its observational nature, the study could not, of course, address the potential underlying mechanisms. However, thanks to it, new questions can be formulated with respect to the progression of the disease.

First, one may ask whether, in addition to the prevalent upper lobe emphysema, other potential mechanisms contribute to accelerate the decline in lung function in smokers. As a matter of fact, FEV1 being a distillate of the physical properties of the lung as well of the airways [18], it would not be surprising that, notwithstanding the clear relationship with upper lobe emphysema, airway remodelling also contributes to worsening the classical spirometric parameters over time. As recently reported by MCDONOUGH et al. [19], gradual narrowing and disappearance of small airways over time is the most typical feature of COPD that precedes the appearance of emphysema and continues afterwards. Thus, it would be of interest to examine how much of the decrease of FEV1 in COPD is caused by small airways disease in addition to emphysema. The problem is not trivial as airway narrowing differently from emphysema can be at least in part pharmacologically controlled. Secondly, it is questioned whether FEV1 is still the primary outcome for followup of the disease. Maximal flow is indeed affected by two major artefacts, i.e. the thoracic gas compression volume [20] and volume history effects of the deep breath [21], with the former having a negative effect on maximal flow by decreasing the absolute lung volume and, thus, the lung elastic recoil, and the latter reducing flow at a given lung volume as a result of a loss of airway-to-parenchyma interdependence. Both cause substantial underestimation of the parameter, especially in emphysema, with peaks well exceeding $50 \%$ in several cases ([20] and unpublished observations), depending on the severity and type of obstructive pulmonary disease. On these grounds, it would be important to know to what extent these artefacts affect the decline of the FEV1 in COPD. Thirdly, the theory that the stretching imposed by breathing on the alveolar surface might facilitate or even dictate the progression of the disease might have important clinical implications. For instance, current guidelines on COPD suggest that the long-acting bronchodilator agents be used only when the FEV1 is $<70 \%$ predicted. Yet, in view of the benefits of therapy on airflow resistance and, thus, pleural pressure gradients and local lung hyperinflation, it may be asked whether administering the bronchoactive medications at earlier stages might be of help to prevent, delay or minimise the occurrence of emphysema. In addition, the mechanical theory would also alert to the possibility that in some patients with prevalent apical emphysema, too intensive rehabilitation programmes might paradoxically deteriorate pulmonary emphysema as a result of the high ventilatory regimes used to improve physical performance and dyspnoea.

In conclusion, if the study by MoHAMED HoEsein et al. [17] brings further support to the notion that emphysema plays a primary role as for the functional progression of COPD, it is the complexity of the disease within a complex organ that needs to drive future research.

\section{STATEMENT OF INTEREST}

None declared.

\section{REFERENCES}

1 Thurlbeck WM, Henderson JA, Fraser RG, et al. Chronic obstructive lung disease. A comparison between clinical, roentgenologic, functional and morphological criteria in chronic bronchitis, emphysema, asthma and bronchiectasis. Medicine (Baltimore) 1970; 49: 81-145.

2 Bergin C, Müller N, Nichols DM, et al. The diagnosis of emphysema. A computed tomographic-pathologic correlation. Am Rev Respir Dis 1986; 133: 541-546.

3 Muller NL, Staples CA, Miller RR, et al. "Density mask". An objective method to quantitate emphysema using computed tomography. Chest 1988; 94: 782-787.

4 Fein AM, Branman SS, Casaburi R, et al. Lung volume reduction surgery. This official statement of the American Thoracic Society was adopted by the ATS Board of Directors, May 1996. Am J Respir Crit Care Med 1996; 154: 1151-1152.

5 International Guidelines for the Selection of Lung Transplant Candidates. Am J Respir Crit Care Med 1998; 158: 335-339.

6 Parr DG, Stoel BC, Stolk J, et al. Validation of computed tomographic lung densitometry for monitoring emphysema in $\alpha_{1}$-antitrypsin deficiency. Thorax 2006; 61: 485-490.

7 Dawkins PA, Dawkins CL, Wood AM, et al. Rate of progression of lung function impairment in $\alpha_{1}$-antitrypsin deficiency. Eur Respir $J$ 2009; 33: 1338-1344.

8 Stolk J, Ng WH, Bakker ME, et al. Correlation between annual change in health status and computer tomography derived lung density in subjects with $\alpha_{1}$-antitrypsin deficiency. Thorax 2003; 58: 1027-1030.

9 Holme J, Stockley RA. CT scan appearance, densitometry, and health status in protease inhibitor SZ $\alpha_{1}$-antitrypsin deficiency. Chest 2009; 136: 1284-1290.

10 Tuder RM, McGrath S, Neptune E. The pathobiological mechanisms of emphysema models: what do they have in common? Pulm Pharmacol Ther 2003; 16: 67-78.

11 Stehbens WE. Proteinase imbalance versus biomechanical stress in pulmonary emphysema. Exp Mol Pathol 2000; 69: 46-62.

12 Mishima M, Hirai, Itoh $\mathrm{H}$, et al. Complexity of terminal airspace geometry assessed by lung computed tomography in normal subjects and patients with chronic obstructive pulmonary disease. Proc Natl Acad Sci USA 1999; 96: 8829-8834.

13 Winkler T, Suki B. Emergent structure-function relations in emphysema and asthma. Crit Rev Biomed Eng 2001; 39: 263-280.

14 West JB. Distribution of mechanical stress in the lung, a possible factor in localisation of pulmonary disease. Lancet 1971; 1: 839-841.

15 Kononov S, Brewer K, Sakai H, et al. Roles of mechanical forces and collagen failure in the development of elastase-induced emphysema. Am J Respir Crit Care Med 2001; 164: 1920-1926. 
16 Gelb AF, McKenna RJ Jr, Brenner M, et al. Lung function 5 yr after lung volume reduction surgery for emphysema. Am J Respir Crit Care Med 2001; 163: 1562-1566.

17 Mohamed Hoesein FAA, van Rikxoort E, van Ginneken B, et al. Computed tomography-quantified emphysema distribution is associated with lung function decline. Eur Respir J 2012; 40: 844-850.

18 Hyatt RE. Forced expiration. In: Macklem PT, Mead J, eds. Handbook of Physiology. Section 3, vol. III, part 1. The respiratory system. Mechanics of breathing. Bethesda, American Physiological Society, 1986; pp. 295-314.
19 McDonough JE, Yuan R, Suzuki M, et al. Small-airway obstruction and emphysema in chronic obstructive pulmonary disease. $N$ Engl J Med 2011; 365: 1567-1575.

20 Sharafkhaneh A, Goodnight-White S, Officer TM, et al. Altered thoracic gas compression contributes to improvement in spirometry with lung volume reduction surgery. Thorax 2005; 60: 288-292.

21 Pellegrino R, Sterk P, Sont JK, et al. Assessing the effect of deep inhalation on airway calibre: a novel approach to lung function in bronchial asthma and COPD. Eur Respir J 1998; 12: 1219-1227. 